\title{
Miscellen.
}

\section{Das Archiv des rheinischen Grafengeschlechtes von Schaesberg in Thannheim.}

$$
\text { Von }
$$

\author{
Archivar G. A. Renz \\ in München.
}

Im gräflichen Schlosse zu Thannheim in Württemberg ruhten seit nahezu einem Säkulum in ganz und gar ungeordneten Zustande; in einer den Anforderungen an einen Archivraum wenig entsprechenden und etwaiger Feuersgefahr durchaus preisgegebenen Bodenkammer in 24 Kästen die verhältnissmässig noch gut erha]tenen und nur zum geringen Theil durch Mäusefrass oder elementare Einflüsse verdorbenen Urkundenschätze des reichsgräflichen Hauses von $\mathrm{Sch}$ a e sberg.

Sie waren zu Anfang unseres Jahrhunderts, theils den Schlössern ehemaliger Herrschaften, theils dem Familienhaus in Diisseldorf entnommen, theils von Kölner und Aachener städtischen und Domkapitel-Archiven extradirt, bier zu einem grossen, jedoch vorerst unbrauchbaren Ganzen vereinigt worden.

$\mathrm{Da}$ fasste vor zwei Jahren der regierende $\mathrm{Gr}$ a f $\mathrm{Hein} \mathrm{ricb}$ von Schaesberg-Than n bim, Herr zu Krieckenbeck und Dillborn den hochherzigen Entschluss, sein an Alter wie Inhalt ausserordentlich werthvolles Haus- und Familienarchiv mit grossem Kostenaufwand einer Neuordnung and Bearbeitung unterziehen zl lassen und mir die Ausführung dieser Arbeit zu übertragen. Nach Ausscheidung der Urkunden von den Akten sah ich, nachdem einerseits wegen Fehlens, anderseits wegen Zugangs zahlreicher Archivalien eine Anlebnung an die noch vorhandenen, alten Repertorien sich nicht mehr thunlich erwies, bei der Neuordnung mich genöthigt, eine von der früheren unabbängige, der Materie sich am besten 
Das Archiv des rheinischen Grafengeschlechtes von Schaesberg in Thannheim. 183

anpassende Trennung und Eintheilung der Dokumente vorzunehmen. Die Urkunden wie die Akten wurden in zwei Hauptabtheilungen geschieden, in I. Ha us- $\mathrm{nd} F$ a milien s a c he n und II. Besitz u $\mathrm{g}$ en, je in alphabetischer Ordnung und chronologischer Reihenfolge. In der ersten Abtheilung nehmen naturgemäss die Schaesbergischen Familiendokumente und die der Agnaten den grössten Raum ein, in der zweiten die Grafschaften $\mathrm{K}$ er p e n L, o m mers u m und Than $\mathrm{m}$ h e i m, worauf ich später noch eingehender zu sprechen komme. Sämmtliche Urkunden wurden jede einzeln in blaue und grüne, die Akten nach ihrer Zusammengehörigkeit faszikelweise in blaue und rothe, mit dem gräflichen Wappen gezierte Umschläge eingelegt, umschniurt und mit aufgelöster Datirung und kurzer Inhaltsangabe versehen, nach Lagerort und durchlaufender Archivnummer signirt, schliesslich ïber den ganzen Urkunden- und Aktencomplex ein neues alphabetisches Generalrepertorium angelegt. Das so neugeordnete Archiv ist jetat im gräflichen Schlusse zu Thannheim in einem gewölbten, trocknen und feuersicheren, an die Rentamtskanzlei anschliessenden Raum des Parterregeschosses untergebracht und die Benützung desselben für Forscherzwecke ermöglicht worden. Es hat der genannte Magnat dadurch der historischen Wissenschaft einen nicht genug anzuerkennenden Dienst geleistet und eine bisher ungekannte, reiche Quelle für die Geschichte des Niederrheins, insbesondere des rheinischen Adels erschlossen.

Ehe ich auf die Besprechung von Umfang und Inhalt des Thannheimer Archivs übergehe, möge mir gestattet sein, einen kurzen Riickblick auf die Geschichte und Entwicklung der reichsgräflich Schaesberg'schen Archive überhaupt zu werfen, wie sich solche aus dem noch vorhandenen Aktenmaterial zusammenfassen lässt. Urspriunglich waren die heutigen Bestandtheile der grätlichen Archive, ohne organische Verbindung, in den verschiedenen Schlössern der rheinischen Besitzungen, die durch Kauf, durch Heirat oder durch Erbschaft an das reichsgräfliche Haus gekommen, aufbewahrt worden, so zu Bernsau, Burga u, Gangelt, Gerdingen, Kerpen, Krieckenbeck, Li chtenberg, Neustadt, Schaesberg, Schoeller u. A., wie aus den noch erhaltenen alten Repertorien hervorgeht. Wegen drohender Kriegsgefahr brachte man 1671 einen Theil der Archivalien, besonders die auf Schloss und Herrlichkeit Schaesberg bezîglichen, nach Ma astrich t za besserer Sicherstellung. Im Jahre 1731 nahmen 
die Brider Johann Wilhelm und Friedrieh Sigis. mund, Reichsgrafen von $S$ ch a es berg eine Erbtheilung vor und es wurde in diesem Vertrag ausdruicklich festgesetyt, dass auch die zu den einzelnen Gütern gehörigen Dokumente getheilt und mit den Scblössern in den Besitz des nenen Inhabers übergehen sollten, nur die eigentlichen Familienpapiere wurden dem älteren Bruder Johann Wilhelm allein zugesprochen, dem jüngeren Bruder mussten aber von den wichtigeren Stïcken beglaubigte Abschriften ausgefolgt werden. Durch diesen Erbvertrag wäre nun allerdings eine bedauerliche Zersplitterung der Schaesbergisehen Archivalien eingetreten, allein bereits 1768 findet sich der gesammte Urkunden- und Aktencomplex wieder in einer Hand, der des kaiserlichen Geheimen Raths, Reichsg rafe $\mathrm{n} \mathrm{A} \mathrm{ugust}$ vo.n Sehaesberg in Düsseldorf vereinigt, nachdem sein Bruder Josef in den Deutschorden eingetreten und Carl Franz Dom. herr zu Münster geworden war. Auf des Grafen August Veranlassung hin wurden sämmtliche Dokumente nach D ii s seld orf gebracht und im dortigen Schaesbergischen Hause in der Kr:inmer-, später Ritterstrasse, Jahrzehnte hindurch in gutem Gewahrsam gehalten. Aus dieser Zeit (ca. 1786) stammen anch die vorhandenen zwei Repertorien, wäbrend ein anderes, nur noch fragmentarisches, wohl bereits um das Ende des 17. Jahrhunderts angelegt worden ist. Auch durch bewirkte Extraditionen der Aachener, Münsterer und Kölner Archive suchte der für sein Familienarchiv sebr besorgte und rührige Reichsgraf August von Schaesberg solches nach Kräften zu vervollständigen. In den unsicheren Zeitläuften zu Ende des vorigen Jahrbunderts, insbesondere nach Beginn der französischen Revolution und der Coalitionskriege, fluchtete man das Archiv nach M ü n ster in das dortige Kapuzinerkloster, wo es bis zum Jahre 1804 verblieb. Erst nach zweijährigen Verhandlungen mit der münsterischen und der preussischen Regierung, sowie dem französischen Tribunal Civil du Departement de la Roer zu Köln gelang es dem Grafen Richard von $S \mathrm{chaesberg}$, die von der letztgenannten Behörde sequestrirten Archivalien im August 1804 wieder ausgefolgt zu bekommen. Graf Richard liess solche unverzüglich nach $\mathrm{S}$ chlos $\mathrm{Th}$ an heim bringen, das ihm als Ersatz fuil die im Lüneviller Frieden zu Verlust gegangenen, linksrheinischen Besitzungen mit dem Kloster Ochsenhausischen Pflegamt Thannheim zu einer Grafschaft unter württembergiseher Oberhoheit vereinigt, zugewiesen worden war. Noch im Jahre 
Dis Archir des rheinischen Grafengeschlechtes von Schaesbergin Thannheim. 185

1815 machte die preussische Regierung wiederholt energiscbe Versllche, einen Theil der rheinisch-preussischen Archivalien extradirt z.ll erhalten, aber Graf Richard war hiezu nicht zu bewegen and so behielten dieselben im Schlosse zu Thannheim bis heute ihren standort und werden ihn dort auch fernerhin behalten.

Anschliessend daran sei noch erwähnt, dass im gräflichen Filialarchivin $\mathrm{Kr}$ ieckenbeck), mit dem ich mich hier nicht weiter zu beschäftigen habe, fast nur Urkunden wnd Akten iiber die Herrschaft Krieckenbeck und ibre Appertinentien liegen; rin der Ordnung des dortigen Archivs entsprechendes, oberflächlich angelegtes und durchgefïhrtes, älteres Repertorium giebt hieriiber weiteren Aufschluss. Durch den Erwerb der Herrlichkeit Krieckenbeck, welche infolge der am 19. Februar $1623 \mathrm{zu}$ Brugoen stattgehabten Vermählung ${ }^{2}$ ) Johan $\mathrm{n}$ Friedrich von Schaesberg mit Ferdinanda von Wachtend o n ck z um B r o i c h, Erbtochter auf Krieckenbeck, an das Haus schaesberg ibergegangen und jeweils der Secundogenitur als Wohnsitz angewiesen war, kam auch das dortige, niemals mit den andern Archivbeständen vereinigte, sondern immer separirte Wachtendonck-Krieckenbeck'sche Archiv in den Besitz der gräflich Schaesbergischen Familie.

Nachdem wir uns nun die historische Entwickelnng; des Schaesbergischen Haus- and Familienarchivs vergegenwärtigt, gehen wir auf den Inhalt desselben über.

Es fanden sich im gesammten 1493 d u r ch w e g u g e druckte Originalurk unden vor, die älteste aus dem Jahre 1275, daran reihen sich 36 des XIV. Jahrhunderts, 252 des XV. und 358 des XVII. Jahrhunderts, der Rest vertheilt sich auf die Zeit von $1600-1825$. In die erste Hauptabtheilung, die der $\mathrm{H}$ a u s- u n $\mathrm{F}$ a mi l i e n s c h en fallen 783, meist Pergamenturkunden, theils in holländischer, theils in niederdeutscher, theils in altfanzösischer Sprache abgefasst. Sie beziehen sich auf nachstehende rheinische Adelsfamilien:

Anstenrode (1463-1577), Barle (1460-1550), Bassenheim (1664), Bex (1610-1673), Benserrade (1429-1501), Belle (14021418), Bernsau (1469-1519), Binsfeld (1495-1681), Blanckart (1569-1633), Bock zum Haen (1709), Bonninghausen (1617-1657),

1) cfr. Burkhardt, Hand- und Adressbuch der deutschen Archive I., 48.

2) Original-Heiratsvertrag im Hauptarchiv zu Thannheim. 
Boulich (1613-1629), Braembach (1502-1587), Brempt (14761613), Burtscheid (1478), Broichhausen (1464-1468), Bylandt (1553), Cobbenrode (1391), Colyn (1573-1739), Cortenbach (14521786), Doersdael (1565), Eclo (1365), Efferen (1644), Eynatten (1477-1738), Elmpt (1674), Ellerborn (1432-1535), Eltze (1483), Esterhazy (1832), Eyll (1650), Fisehenich (1469-1562), Freytag (1555), Gaelen (1424), Gymnich (1513), Hanxler (1614-1665), Hatzfeld (1574-1586), Heerde (1484), Hembach gen. Hoen (14691580), Hesscheide (1382), Horion-Hochkirchen (1563), Holsit (1524), Holthausen (1450-1604), Holtmoelen (1448), Hompesch (1495), Horst (1541-1707), Jülich-Berg-Cleve (1418--1450), Kessel (1462 -1527), Ketzgen (1676-1691), Landsberg. (1404-1571), Lichten. berg (1293), Lotzbeck (1790), Luninck (1450-1631), Lützenrode (1524-1615), Lyntzenich (1566), Manderscheidt (1668), Marckelsbach gen. van Alver (1520), Merode (1527-1705), Merveldt (1763), Metternich zur Gracht (1614-1790), Mir (1443-1494), Munckert (1438-1480), Munkenbicke van der (1347), Nassau, Junggraf von (1480-1487), Nesselrode (1453-1738), Neuenbeim (1455), Neuhoff gen. Ley (1491-1738), Plettenberg (1530-1634), Portzen von der (1673), Quadt (1554-1624), Randerath (1693), Roese (1443), Schaloen, Schalnyn (1545-1605), Schaesberg (1469-1840), Schoeller (1539-1697), Spee (1562), Spreuwart (1528-1530), Sayn-Wittgenstein (1488), Stail van Holstein (1465 - 1570), Strithagen (1434-1491), Vavesbeich (1402), Veels van dem (1427-1433), Velbrück (1702), Wachtendonck (1455-1696), Waldtoss (1599-1656), Weerst.(1476 -1568), Wenge (1778), Westerholt-Lembeck (1641-1756), Weyenhorst, Wittenhorst (1527-1594), Wylich-Bernsau-Combach (1596 -1697) und Zwivell (1468-1511).

Die zweite Hauptabtheilung, die der B e s it z un ge n, umschliesst in 710 Pergamenturkunden folgende Gïter und Herrscbaften ${ }^{1}$ ): Aachen, Weisse Frauen (1479), Dominikanerinnen (1482 -1500), Averbeeden, Dominikanerkloster (1304), Acher, Fischerei (1463-1507), Kloster Altenberg (1374-1523), Benzenrade (13611782), Boessenich, Dominikanerinnen (1486), Burgau (1710), Coenraedt (1651-1672), Düren, Stadt (1631-1773), Düsselbach, Fischerei $(1390-1431)$, Duisseldorf, Collegiatstift $\left.(1555)^{2}\right)$, Heiligkreuz-

1) Es sind nur die umfangreicheren Besitztitel herausgezogen.

2) Der Inhalt dieser nicht uninteressanten Urkunde ist folgender: 1555. Februar 14. Decan und Capitel der Collegiatkirche zu Diisseldorf bestä- 
Das Archiv des rheinischen Grafengeschlechtes von Schaesberg in Thannheim. 187

britider (1600) Dïsseldorf, Schaesbergisches Haus (1563-1804), Escherode (1425-1436), Elver-Forst (1373-1375), Erckelens (1711), Fischenich (1540-1542), Gangelt (1511), Genna-Letmate (14851596), Gerdingen (1431-1774), Goldberger-Höfe (1550-1719), Haenrode (1431-1553), Haerbeck (1465-1556), Hardenberg (1484), Harzhof-Wipperfurde (1449-1450), Hemmersbach (1481-1738), Heer $(1446-1450)$, Herle (1546-1588), Hoeve-Dalheim (14811491), Kerpen (1284-1792), Kessenich (1700-1784), Kevernberg (1455-1558), Kintzweiler-Hahn (1701-1726), Köln, Erzstift (14141577), Köln, Schaesbergisches Haus (1584), Lichtenberg ${ }^{1}$ ) (14621750), Linzenich (1338-1491), Loevenich (1383-1504), Maastricht, Stadt (1429-1620) 22 Urkunden, Neustadt (1512-1559), Olpe, Schloss (1383), Overath (1430-1454), Ryndorp (1438-1471), Saffenhurg (1708), Schaesberg, Schloss und Herrlichkeit (1581-1791), schimmershof (1447-1659), Schoeler (1372-1802), ThannheimOchsenhausen, Benediktinerkloster in Württemberg (1307-1800), Valckenburg, Faucquemont (1397-1500), Weisweiler (1436-1444), Werden, Kaiserswörth (1275-1367), Weyer (1427-1725), Wildenburg (1600-1706) und Wyek bei Maastricht (1499-1522).

Dieser Urkundencomplex enthält $14 \mathrm{~K}$ a i s e rurk unde $\mathrm{n}$, die älteste von $\mathrm{Rudolf}$ von $\mathrm{Habsburg}$ d. d. 1284, Febrnar 11. Estein, ein Privileg für die Herrschaft K e r p e n (Copie). Maximilians I. d. d. 1510, Mai 27. zu Worms und andere; weiterhin $12 \mathrm{k} \ddot{0} \mathrm{nigl}$. $\mathrm{span}$ is che des 16. und 17. Jahrhunderts, 52 herzogl. Jïl i ch-Cleve-Bergische, 5 päpstli che, dann eine ganze Serie bischöfliche, speziell $\mathrm{k} \ddot{0} \mathrm{l} n$ is $\mathrm{ch}$ e Urkunden. Es findet sich im Schaesbergischen Archive historisches Material über das Herzogthum Brabant, die Grafschaft Valckenburg, den Deutschorden, über die Städte: Aachen, Dortmund, Diissel-

tigen, dass ihnen von den Verordneten des Ausschusses der Ritterschaft im Fürstenthum Berg alle Briefe und Siegel der Privilegien der Ritterschaft und Unterthanen dieser Fürstenthümer, wie auch der Herrschaften Blanckenberg und Lewenberg in einer eisenbeschlagenen Kiste und ebenso alle Copien ron denselben und andern Schriften in einer weiteren Kiste in Gewahrsam gegeben und sie solcbe auch angenommen haben. Siegler: Das Capitel der Düsseldorfer Collegialkirche. (Orig. Perg. Siegel ab.)

1) In der 1895 erschienenen Schrift: "Succession généalogique des Seigneurs de Lichtenberg publié par Lou is Baron de Crassier dans les publications du Limbourg (Sep-Abdr. Maastricht 1896) ist bedauerlicherweise das Schaesbergische Archiv nicht herangezogen. 
dorf, Limburg, Liittich, Maastricht; die Domkapitel: Hildesheim, Köln, Lüttich, Münster, Paderborn, Roermond, Speyer; die Klöster und Stifte: Aachen, Dominikaner, Weisse Frauen; Averbeeden, Dominikaner; Cöln, St. Caecilien, St. Cunibert, St. Gereon, St. Marien in Capitolio, St. Pantaleon; St. Corneli-Münster; Corvey; Dal. heim; Düsseldorf, Carmeliten und Heiligkrenzbrüder; Elsen; Greve. rade; Hoven, Cistercienserinnen; Kaiserswörtl; Lichtenberg, Collec. ten; Lüttich, St. Martin; Naastricht, B. Marie Virg., Minoriten, St. Servatius; Neuss, St. Quirin; Stavelot; Steinfelden und Syberg. Alle diese Namen figuriren in den Urkunden, theils als Aussteller, theils als Empfänger, theils als Siegler.

Was nun das reichhaltige A k te n mate ria 1 betrifft - es umfasst 2367 Faszikel, mebrfach iiber das Jahr 1500 binaufreichend -, so wollen wir uns auf die Angabe einiger, wichtigerer Abtheilungen beschränken. Ein grosses Contingent stellen Prozessakten, Gerichtsprotocolle, Kriegssachen (30 jäbriger Krieg, österreich. Erbfolgestreit, siebenjähriger und napoleonische Kriege), Rentregister, Amts- und Kellerei-Rechnungen der Grafschaften Kerpen-Lommersum von $1581 \mathrm{ab}$, und Thannheim 17. Jahrhundert; der Herrichkeiten Gerdingen-Neustadt von $1511 \mathrm{ab}$, Lichtenberg von 1464 ab, Merzenich, Overath, Reinardstein von 1343 ab, Schaesberg von $1500 \mathrm{ab}$, Schoeller von $1482 \mathrm{ab}$, Weyer von $1584 \mathrm{ab}$ und Wildenburg von 1600 ab. Ausserdem seien von den Alienis noch hervorgehoben: ein Schuld- und Wiederschuldbuch Diderich's von Agrys ${ }^{1}$ ), vom 10. Dezember 1595 anfangend; Bergisches Ritterrecht d. d. 10. Juni 1463 (Copie); Amtssachen von den churpfälzischen Aemtern Barmen und Beyenburg, Blankenberg, Bruggen und Dahlen; Rentregister des Laethofes van Fee im Kirchspiel von Kanne von 1481; Rentregister der Junker Johann und Hermann van Eynatten iiber denselben Lehenhof 1528; Rechnungen siber französische Contributionen für das Oberquartier Gelderland 1672 ff., 3 Gerichtsentscheide über die zur Herrlichkeit Neustadt gehörige Jurisdiktion von fünf in der Stadt Brée gelegenen Hänsern 1542-1552, Amtsangelegenheiten und Rechnungen des St. Huber-

1) Auf der Rückseite des Pergamentumschlages steht unter anderem "Dedereych vonn Agrys beinn ich genandt, Mein Sach stell ych in Gotteshandt" und „Hoffenn und Harrenn,

Macht manchenn zum Narrenn". a. d. 1595. 
Das d rchiv des rheinischen Grafengeschlechtes von Schaesberg in Thannheim. 189 tusordens 1708-1710 und $1711 \mathrm{ff}$., und I. Rechnung der St. Hubertusordens-Kasse, 1. Oktober 1708-1710 30 September; gedruckte, cliurpfälzische Information über die Geschichte der Grafschaften herpen-Lommersum; Verzeichniss der Lommersheimer Mann- oder Lebenguiter 1581-1645; Rechnungen der St. Servatiuskirche zu Jaastricht, angelegt vom Canonicus Olivier van Eynatten, 15851595, und 1595-1596; Fragment eines Copialbuches versehiedener tdelsfamilien 1544 ff.; Anszug aus dem Lehenregister der Abtei Stavelot iiber die Herrlichkeiten Reinardstein und Polsuyr 16. saec.; genealugrische Notizen über die Familien von Schoeler und Quadt 1535ff.; tnitsangelegenheiten Rutgers von Schoeler, als Amtmann von Mettman 1579; Rechnungen Friedrichs von Schaesberg über Einnabmen und Ausgaben des Landes von Valkenburg 1612-1615; Register iiber die Landlasten von Valckenburg und den darin gelegenen adeligen Häusern und Gütern 1558-1612; Ausgabenbuch Diderichs ran Weerst der beim Ableben und Begräbniss seiner Mutter gehabten Unkosten d. d. 1532, Septbr. 24; 4 Faszikel westtälische kireistagrs-Protokolle 1718; 2 Faszikel westfälische ReichsgrafenCollegialakten $1740-1790 \mathrm{ff}$; 3 Faszikel niederreinisch-westfälische Lireissachen $1770 \mathrm{ff}$; Inventur iiber die im Sterbebause Ulrichs van Weerst vorgefundenen Briefschaften, Register etc. 1538; weiterhin mögen noch erwäbnt sein: eine alte, churpfälzische Burgverordnungr 1541; über 150 Faszikel churpfälzische Hofkammerakten, vom 16.-18. Jabrbundert, Bauten, Kasernen, Klöster, Kriegssachen, Ritterschaftsangelegenheiten u. A. betreffend; Correspondenzen zwischen Hessen-Darmstadt und Churpfalz in Postangelegenbeiten (1705); Ueberweisung von Deputatgeldern an die Churfiurstin Maria Anna von der Pfalz nach Florenz und deren Briefe an den Reichsgrafen Johann Wilhelm von Schaesberg aus den Jahren 1719-1742; Stammbäıme und Aufschwörungen einer grossen Anzahl rbeiniseher Adelsfamilien von $1313 \mathrm{ab}$; und schliesslieh von den zahlreichen, besonders Cortenbach, Plettenberg, Schaesberg, Schoeler und Wylich'schen Processakten, solche der Klöster St. Quirin in Neuss 1678 ff., Steinfelden 1631 ff., Königsdorf 1698 ff., Jesuiten in Bonn 1716, Serviten in Bonn $1717 \mathrm{ff}$. und die beiden ältesten Processakten zwischen den Bridern von Lünninck und Neuhoff gen. Ley wegen des Hauses Gorghausen $1449 \mathrm{ff}$. und dem Freiherrn von Schaesberg contra Melchior von Cortenbachs Erben beim Hofe zu Brabant wegen der Güter zu Coenraedt $1577 \mathrm{fff}$. Aus obigem, thunlichst kurzgefasstem Bericht erhellt zur Genüge, 
190 G. A. Renz, Das Archiv des rhein. Grafengeschlechtes von Schaesberg etc.

dass das gräflich von Schaesbergische Haus- und Familienarchiv eine reiche Fundgrube für Forschungen auf dem Gebiete rbeinischer Adels-, Kloster- und Städtegeschichte bildet, die Dank der Opfer. willigkeit des jetztregierenden Graten nunmehr sich aufgethan. Eine Publikation der ältesten und wichtigsten Urkunden des Schaesbergischen Hauptarchivs wird vielleicht im Laufe dieses Jabres nachfolgen.

\section{Zwei Steinfelder Urkunden der Kölner Erzbischöfe Konrad und Siegfried.}

Mitgetheilt von

Dr. Armin Tille (Bonn).

Die beiden im Folgenden veröffentlichten Urkunden entstammen dem Archiv des Klosters Steinfeld und sind bisher unbekann. geblieben.

I. In der ersten spricht Erzbischof Konrad von seinen Kriegen und namentlich von der Befestigung der Stadt Ahrweiler, welche also 1260, wenn sie nicht vollendet ist, so doch $\mathrm{zu}$ einem gewissen Abschluss gekommen sein muss. Das Kloster Steinfeld hat ihn materiell dabei unterstützt, und zur Entschädigung wird es nunmehr von Zoll und Accise zu Abrweiler befreit. Die Accise liegt bereits in den Händen der Stadt. Dieses städtische Recht ist hier also früher ausgebildet als bei den niederrheinischen Territorialstädten: nur Wesel erwirbt das Recht der Acciseerhebung noch im 13. Jahrh. (1278)1), während Hüssen 1348, Uedem 1359, Griethausen 1374,Emmerich 1377 und Dinslaken gar erst 1420 folgen. Auch in Bonn wird erst 1318 der Stadt eine zeitlich bescbränkte (12jährige) Accise verliehen ${ }^{2}$ ). Der Erzbischof als Landesherr nimmt das Recht, von der Accise zu befreien, für sich in Anspruch

1) Vgl. Liesegang, Niederrheinisches Städtewesen, S. 75 und ferner $17 \pm, 185,190,207,271$.

2) Vgl. Tille, Uebersichten über den Inhalt der kleineren Archire, S. 134, Nr.900. Ueber das landesherrliche Recht, Zoll und Accise zu erheben vgl. Lacomblet, Urkb. III, Nr. 326; 1338, Mai 10. Kaiser Ludwig gesteht dem Markgrafen von Jülich dieses Recht in allen Orten seines Gebietes zu. 